\title{
NLSE-based model of a random distributed feedback fiber laser
}

\author{
Sergey V. Smirnov*a,b, Dmitry V. Churkin ${ }^{\mathrm{a}, \mathrm{b}, \mathrm{c}}$ \\ ${ }^{a}$ Novosibirsk State University, 630090, Novosibirsk, Russia; ${ }^{b}$ Aston Institute of Photonic \\ Technologies, Aston University, Birmingham, B4 7ET, United Kingdom; ${ }^{\mathrm{c}}$ Institute of Automation \\ and Electrometry SB RAS, 1 Ac. Koptyug ave., Novosibirsk, 630090, Russia
}

\begin{abstract}
In this work we propose a NLSE-based model of power and spectral properties of the random distributed feedback (DFB) fiber laser. The model is based on coupled set of non-linear Schrödinger equations for pump and Stokes waves with the distributed feedback due to Rayleigh scattering. The model considers random backscattering via its average strength, i.e. we assume that the feedback is incoherent. In addition, this allows us to speed up simulations sufficiently (up to several orders of magnitude). We found that the model of the incoherent feedback predicts the smooth and narrow (comparing with the gain spectral profile) generation spectrum in the random DFB fiber laser. The model allows one to optimize the random laser generation spectrum width varying the dispersion and nonlinearity values: we found, that the high dispersion and low nonlinearity results in narrower spectrum that could be interpreted as four-wave mixing between different spectral components in the quasi-mode-less spectrum of the random laser under study could play an important role in the spectrum formation. Note that the physical mechanism of the random DFB fiber laser formation and broadening is not identified yet. We investigate temporal and statistical properties of the random DFB fiber laser dynamics. Interestingly, we found that the intensity statistics is not Gaussian. The intensity auto-correlation function also reveals that correlations do exist. The possibility to optimize the system parameters to enhance the observed intrinsic spectral correlations to further potentially achieved pulsed (mode-locked) operation of the mode-less random distributed feedback fiber laser is discussed.
\end{abstract}

Keywords: Random fibre laser, Rayleigh scattering, NLSE.

\section{INTRODUCTION}

In past few years, a new type of fibre laser - a random distributed feedback fibre laser - is widely investigated ${ }^{1,2}$. The laser operates via extremely weak random scattering owing to random Rayleigh backscattering (RS). The gain is provided by a stimulated Raman scattering. Up to date, a number of different random DFB fiber lasers schemes is realized $^{3-17}$. Namely, random fibre lasers can operate in different spectral bands ${ }^{3,4}$, be cascaded, i.e. emit higher order Stokes waves ${ }^{3-5}$, the laser can be easily tunable ${ }^{13,14}$, provides multi-wavelength output ${ }^{6,10,12}$. The noise level of random DFB fiber lasers could be lower than of conventional lasers ${ }^{18}$ making them attractive for telecom applications. Random DFB fiber lasers are also applied for sensor applications ${ }^{19-21}$.

A theoretical description of random lasers is challenging in general, see, for example, review ${ }^{22}$. In the field of random DFB fibre lasers, the latest achievements in the field of description of random DFB fibre laser properties are following. A simple power balance model ${ }^{1,17}$ provides a good description of power performances of the random DFB fibre laser including the generation threshold, the longitudinal generation power distribution ${ }^{17}$. The laser could be optimized using the power balance model ${ }^{23}$ as well as noise properties could be considered ${ }^{18}$. To describe spectral and temporal properties, other models are needed. For example, the random generation could be represented as sets of modes either of passive or active cavity, localized or extended ${ }^{24-28}$. Other approaches based on Maxwell's equations combined with the rate equations of a n-level system do exist ${ }^{29,30}$. Numerical methods to solve such systems are varying and include MonteCarlo simulation of a random walk of photons ${ }^{31}$, the finite difference time domain method ${ }^{32-34}$, the transfer matrix method $^{35,36}$. Despite a number of methods available to describe spectral and temporal properties of random lasers, none of them are not applied to the description of the random DFB fiber laser. In general, its spectral, temporal and statistical properties were not studied theoretically or numerically until very recently.

*sergeyv.smirnov@gmail.com.

Nonlinear Optics and Its Applications VIII; and Quantum Optics III, edited by Benjamin J. Eggleton, Alexander L. Gaeta, Neil G. R. Broderick, Alexander V. Sergienko, Arno Rauschenbeutel, Thomas Durt, Proc. of SPIE Vol. 9136, 91361P · C 2014 SPIE · CCC code: 0277-786X/14/\$18 · doi: 10.1117/12.2052232 
Note that the spectral properties of the random DFB fibre lasers have not been yet studied systematically in experiments. There are only few attempts. As an example of some spectral studies, both power and spectral properties of the forwardand backward-pumped random DFB fibre lasers were measured depending on the ratio between the backward- and the forward-propagating pump wave powers $\mathrm{in}^{8,9}$. It was found that the spectral broadening was more pronounced in the forward-pumped configuration resulting in a $0.9 \sim \mathrm{nm}$ spectral width for $70 \sim \mathrm{mW}$ of the output power. The authors suggested a difference in the longitudinal power distributions as the origin of deviations in the random lasers behavior.

In the recent paper $^{37}$, we applied a NLSE-based model to describe numerically power, spectral, temporal and statistical properties of the random DFB fiber laser radiation. In the present manuscript, we describe in details the simple NLSEbased model applied for a random DFB fiber laser. To do that we take into account an average energy feedback via random Rayleigh backscattering.

\section{NLSE-BASED NUMERICAL MODEL OF THE RANDOM DFB FIBRE LASER}

In general, NLSE-based model is the most powerful and widely applied method to describe various fiber optics systems $^{38}$. In particular, NLSE-based modeling describes well power, spectral, temporal and statistical properties of quasi-CW fiber lasers with conventional cavities made of point-based mirrors including Brillouin lasers ${ }^{39}$, Ytterbitumdoped fiber lasers ${ }^{40,41}$ and Raman fiber lasers (RFLs) ${ }^{42-45}$. We use almost the same model as in ${ }^{42-45}$ except the term accounting for the random feedback.



Figure 1. The considered setup.

Here we consider numerically the following random DFB fibre laser scheme, Fig. 1. We consider the laser of length 41 $\mathrm{km}$ pumped at $1455 \mathrm{~nm}$ by a quasi-CW pump laser (the pump radiation is also governed by NLSEs). In the considered scheme, the highly reflective broadband mirror (centered at $1555 \mathrm{~nm}$ ) is used at one fibre end. Note that this configuration is equivalent to the configuration with two pump lasers, doubled fibre length and no any mirror in the cavity because of symmetry. Because of the Raman gain, the laser generates near $1555 \mathrm{~nm}$. The equation set is following:

$$
\begin{aligned}
& \frac{\partial A_{p}^{+}}{\partial z^{ \pm}}-\frac{1}{v_{g s}} \frac{\partial A_{p}^{+}}{\partial t}+\frac{i}{2} \beta_{2 p} \frac{\partial^{2} A_{p}^{+}}{\partial t^{2}}+\frac{\alpha_{p}}{2} A_{p}^{+}=i \gamma_{p}\left|A_{p}^{+}\right|^{2} A_{p}^{+}-\frac{g_{p}(\omega)}{2}\left(\left\langle\left|A_{s}^{ \pm}\right|^{2}\right\rangle+\left\langle\left|A_{s}^{\mp}\right|^{2}\right\rangle\right) A_{p}^{+} \\
& \frac{\partial A_{s}^{ \pm}}{\partial z^{ \pm}}+\frac{i}{2} \beta_{2 s} \frac{\partial^{2} A_{s}^{ \pm}}{\partial t^{2}}+\frac{\alpha_{s}}{2} A_{s}^{ \pm}-\frac{\Delta A_{s}^{\text {Rayleigh }}}{\Delta z}=i \gamma_{s}\left|A_{s}^{ \pm}\right|^{2} A_{s}^{ \pm}+\frac{g_{s}(\omega)}{2}\left\langle\left|A_{p}^{+}\right|^{2}\right\rangle A_{s}^{ \pm}
\end{aligned}
$$

Here $A$ is complex field envelope, $t$ is a time in a frame of references moving with pump, $\mathrm{v}_{\mathrm{gs}}$ is a difference between pump and generation Stokes waves inverse group velocities, $\beta_{2}, \alpha, \gamma, g$ are dispersion, linear attenuation, Kerr and Raman coefficients, $\omega$ is the frequency detuning from the center of the gain profile, $L$ is the laser total length, $\Delta \mathrm{A}_{\mathrm{s}}^{\text {Rayleigh }}$ is given by Eq. (3). Indices "+" and "-" denote generated waves co- and counter-propagating with the pump wave. Here we define the longitudinal coordinate for the generation wave $z_{ \pm}$as $z=0$ at a starting point of the generation wave propagation (either $\mathrm{A}_{\mathrm{s}}^{+}$or $\mathrm{A}_{\mathrm{s}}^{-}$) and $\mathrm{z}=\mathrm{L}$ at the final point of the propagation, i.e. "+" wave has a coordinate $\mathrm{z}_{+}$while propagating, and "-" wave has a coordinate z., and both waves propagates in the positive direction of $z$-axis. At the same time, the longitudinal coordinate value $\mathrm{z}_{+}$for the co-propagating Stokes wave $\mathrm{A}_{\mathrm{s}}^{+}$corresponds to the value of the longitudinal coordinate $\mathrm{z}_{-}=\mathrm{L}-\mathrm{Z}_{+}$for the counter-propagating Stokes wave $\mathrm{A}_{\mathrm{s}}^{-}$and vice versa. Equations are $\mathrm{z}$-averaged over the dispersion walk-off length of the generation and pump waves, thus the phase in cross-modulation term is zero ${ }^{45}$. 
White noise as an initial condition is used to take into account the spontaneous Raman scattering ${ }^{46}$. Raman gain is approximated by the parabola, $\mathrm{g}_{\mathrm{i}}(\omega)=\mathrm{g}_{\mathrm{i}}-\mathrm{k} \omega^{2}$, where $\mathrm{k}=0.0062 \mathrm{ps}^{2}(\mathrm{~W} \mathrm{~km})^{-1}, \mathrm{i}=\mathrm{s}, \mathrm{p}$. The equations are integrated along $\mathrm{z}$ using an iterative approach with an integration step $\Delta \mathrm{z}$, i.e. when integrating equations for $\mathrm{A}_{\mathrm{s}}^{+}$, values $\mathrm{A}_{\mathrm{s}}^{-}$obtained on previous iteration are used, and vice versa.

We use following parameters: $\alpha_{\mathrm{s}}=0.046 \mathrm{~km}^{-1}, \alpha_{\mathrm{p}}=0.055 \mathrm{~km}^{-1}, \gamma_{\mathrm{s}}=1.09(\mathrm{~km} * \mathrm{~W})^{-1}, \gamma_{\mathrm{p}}=1.31(\mathrm{~km} * \mathrm{~W})^{-1}, \mathrm{~g}_{\mathrm{s}}=0.36$ $(\mathrm{km} * \mathrm{~W})^{-1}, \mathrm{~g}_{\mathrm{p}}=0.39(\mathrm{~km} * \mathrm{~W})^{-1}, \beta_{2 \mathrm{~s}}=20 \mathrm{ps}^{2} / \mathrm{km}, \beta_{2 \mathrm{p}}=35 \mathrm{ps}^{2} / \mathrm{km}, 1 / \mathrm{v}_{\mathrm{gs}}=-2.3 \mathrm{~ns} / \mathrm{km}, \mathrm{L}=370 \mathrm{~m}$, pump power $\mathrm{P}=3 \mathrm{~W}$. Laser mirrors were modeled by supergaussian fiber Bragg gratings (FBGs) of $0.5 \mathrm{~nm}$ width.

The Rayleigh backscattering feedback is taken into account via term $\Delta \mathrm{A}_{\mathrm{s}}{ }^{ \pm \text {Rayleigh }}$ defined as:

$$
\Delta A_{s}^{+ \text {Raylegh }}=\left(\varepsilon \cdot \Delta z \cdot \frac{\int_{-\infty}^{+\infty} \mathrm{d} \omega \mid A_{s}^{-}\left(L-z^{+}\right)^{2}}{\int \mathrm{d} \omega \mid A_{s}^{-}\left(L-z_{\text {prox }}^{+}\right)^{2}}\right)^{1 / 2} \cdot A_{s}^{-}\left(L-z_{\text {prox }}^{+}\right) \cdot e^{i \varphi_{0}+i \omega \tau_{0}}
$$

Here only the energy income from the Rayleigh backscattering is taken into account via term $\Delta \mathrm{A}_{\mathrm{s}}{ }^{ \pm \text {Rayleigh }}$ similarly to the balance equation set. Taking into account only an average energy income results in good quantitative description of a random DFB fiber laser power performance within the power balance model ${ }^{10,23}$. The same approach is used to deal with RS in amplifiers ${ }^{48}$. We follow this approach and add an average term proportional to $\varepsilon=4.5 \times 10^{-5} \mathrm{~km}^{-1}$ to Eq. (2) (this value may vary depending on fiber NA and fabrication method $^{49}$ ). At the same time, the generation wave depletion due to the Rayleigh backscattering is considered through the linear losses $\alpha$. Rayleigh scattering induced energy income to the pump wave is neglected as it is not amplified. A random phase factor exp $\left(i \phi_{0}+i \omega \tau_{0}\right)$ with a random phase $\phi_{0}$ and time $\tau_{0}$ shifts is used. We do not take into account correlation properties of the random backscattering ${ }^{50}$. As the Rayleigh term includes the optical spectrum of the counter-propagating wave, $A_{s}^{\mp \text { Raylegh }}(\omega)$, one needs to save optical spectra at each integration step, which is technically impossible. To deal with that, the optical spectra of the generation waves are saved only at very limited number of points along $\mathrm{z}$ coordinate at each iteration (at $\mathrm{N}=50 \mathrm{z}$-points in the present case). That means $Z_{\text {prox }}(z)$ is a staircase function which approximates $\mathrm{z}$ with a set of $\mathrm{N}=50$ steps along $\mathrm{z}$, each of them is a $\mathrm{z}$ coordinate of the closest point where the spectra of counter-propagating wave is saved at the previous iteration.

\section{SPECTRAL AND TEMPORAL PROPERTIES OF THE RANDOM DFB FIBRE LASER}

We start our consideration from the calculation of the random DFB fibre laser output power as a function of the pump power. The simulation predicts a lasing threshold close to $0.8 \mathrm{~W}$ in agreement with experimental observations ${ }^{1,2}$ and analytical calculations ${ }^{1,23}$. Well above the threshold, the output power grows linearly with pump power that also agrees well with previous experimental observations ${ }^{1,2}$. The numerically calculated within NLSE-based model power distributions agree qualitatively well with experiment and analytical calculations made within power balance equations ${ }^{17}$.
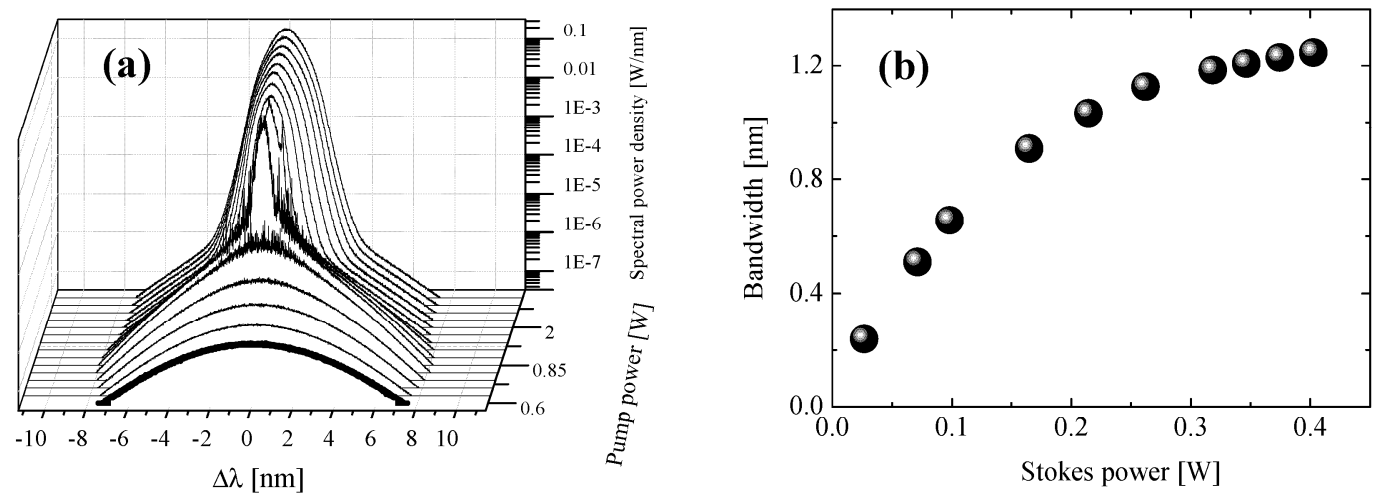

Figure 2. (a) The generation spectrum over the pump power (b) The spectrum rms width depending on the generation power. 

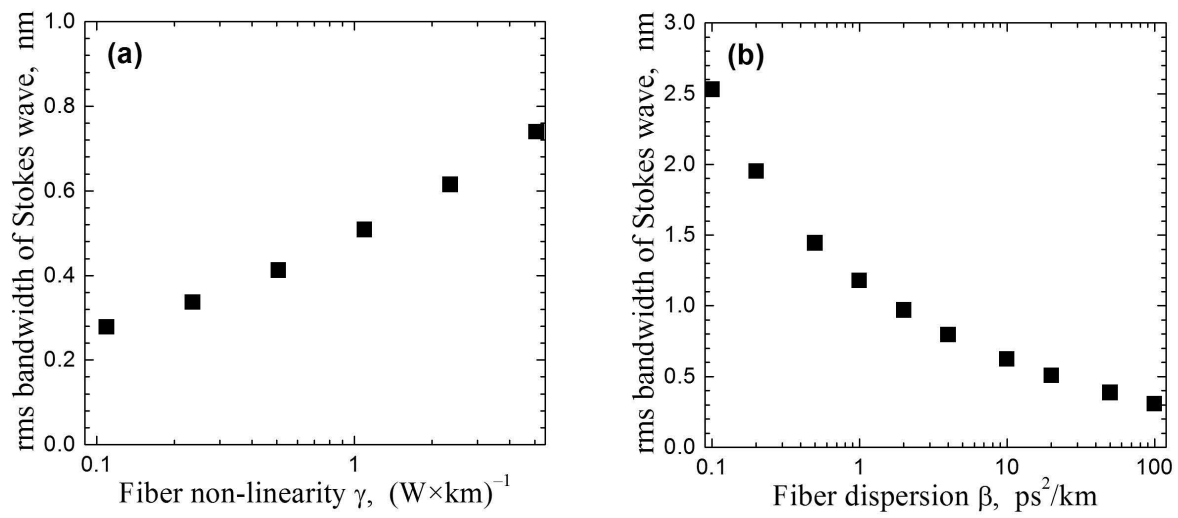

Figure 3. The spectrum broadening at different dispersion (a) and nonlinearity (b) values. Pump power $\mathrm{P}=2 \mathrm{~W}$.

Typical averaged spectra of generated Stokes wave are shown in Fig. 2a. As in experiments, the calculated generation spectrum becomes narrower than the amplified spontaneous emission profile while the pump power increases over the generation threshold indicating that the real lasing is achieved. Note that at later stages of evolution, the spectrum becomes broader with the power, Fig. 2b. Similar nonlinear spectral broadening is observed in some experiments too. We anticipate that such spectral broadening is owing to Kerr nonlinearity and dispersion interplay similar to the processes in the conventional mirror-based fiber lasers ${ }^{52-54}$. Thus a simple model taking only an average random Rayeligh backscattering strength provides a good description of both power and spectral properties of random DFB fiber laser.

In the random DFB fibre laser the spectrum broadening law depends strongly on the fibre parameters. Indeed, in our system changing the nonlinearity at fixed pump power and fixed dispersion, we observe that spectrum becomes broader in the systems with larger nonlinearity, Fig. 3a. At the same time, the generation spectrum becomes narrower for systems with higher dispersion, Fig. 3a, if the pump power and nonlinear coefficients are fixed. This could be understood as dispersion prevents to different spectral components to interact nonlinearly in effective way. There are no up to date any experimental data to compare our findings of dispersion and nonlinearity influence on spectrum width. The proper dispersion and/or nonlinear management could be a practical tool to change the spectral properties in real random DFB fiber laser systems.

One more important property which could be calculated within the developed model is the spectrum evolution over the fibre, Fig. 4. The spectrum has a same spectral shape in all points over the fiber which proves the fact the real laser nature of the generation.

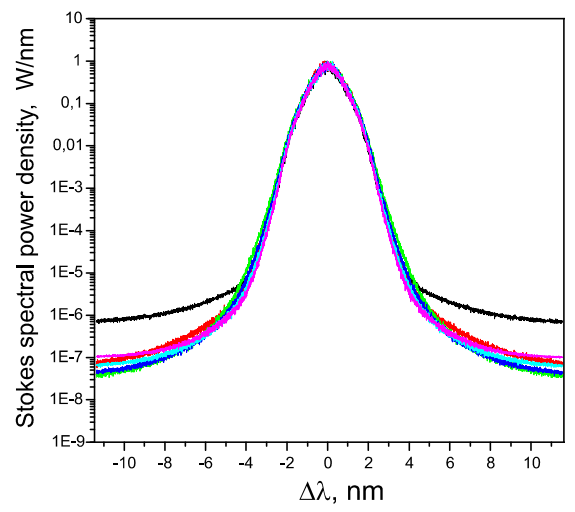

Figure 4. The generation spectrum evolution along the fiber: the spectrum generated at different points along the resonator is shown. The all fiber length is devided by 50 equally spaced points.

Temporal and statistical properties of the random DFB fibre laser radiation could also be calculated within the NLSEbased model. The intensity dynamics reveals highly stochastic nature of the radiation, Fig. 5a. The typical time scale of 
fluctuations is $\sim 5 \mathrm{ps}$ as it is revealed by the intensity autocorrelation function, Fig. $5 \mathrm{~b}$. Note that real-time oscilloscope are limited in bandwidth to $33-60 \mathrm{GHz}$, so the measured intensity dynamics will be averaged, Fig. 5a.
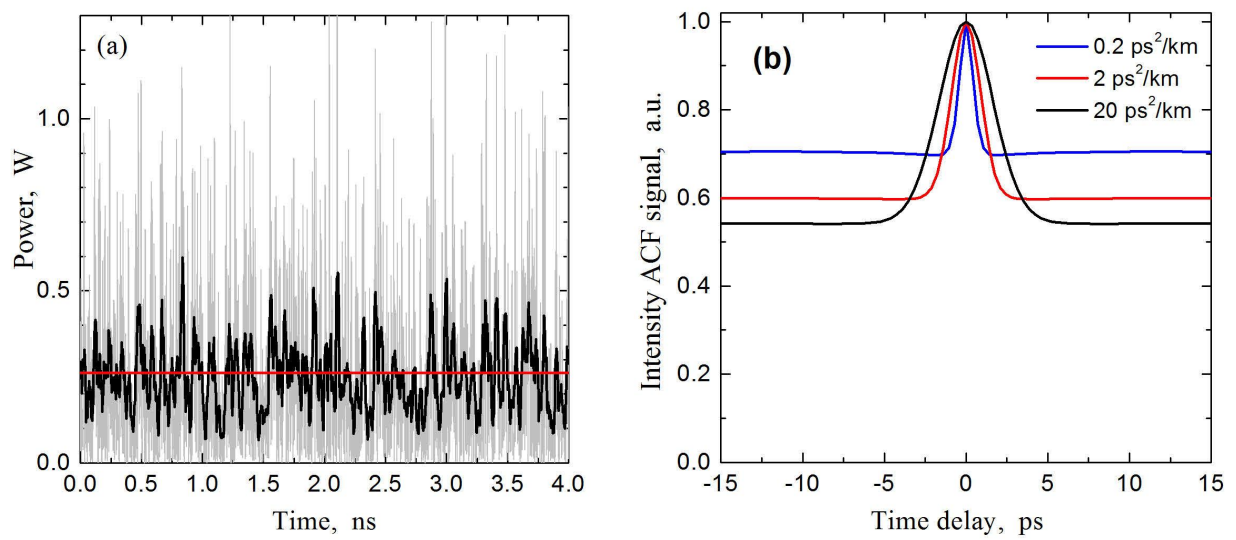

Figure 5. (a) Typical intensity dynamics (grey shows original simulated data, black - smoothed with a bandwidth of $33 \mathrm{GHz}$, red - average lasing power level), (b) Intensity ACF Pump power is $2 \mathrm{~W}$ on all graphs.

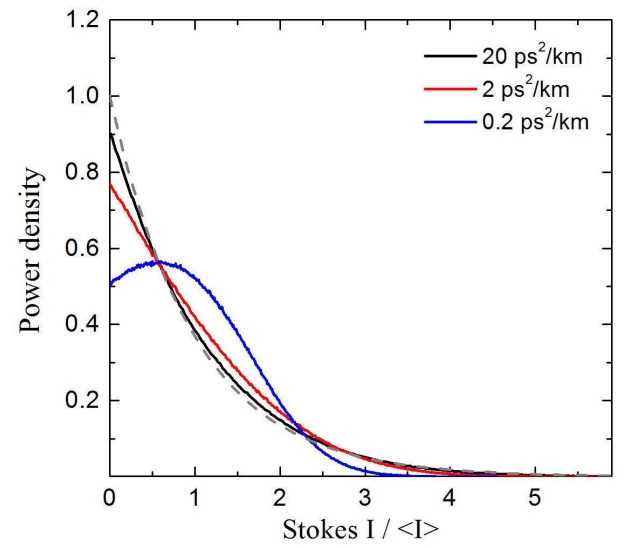

Figure 6. Intensity statistics at different dispersion value. The grey dashed curve corresponds to the exponential statistics of uncorrelated modes.

The more intriguing question is the radiation statistics. We found that the intensity statistics is not completely Gaussian in random DFB fiber laser. The lower the dispersion, the more non-exponential is intensity probability density function (pdf), Fig. 4(c), revealing correlations in radiation. The existence of correlations could be also revealed in the intensity autocorrelation function, which level is higher than 0.5 expected to be in the case of stochastic radiation, Fig. $5 \mathrm{~b}$. The intriguing question of non-gaussian intensity statistics in the radiation of the random DFB fiber laser has to be further investigated. Note that the non-gaussian intensity statistics is previously reported in conventional mirror based laser cavities ${ }^{41-45}$. In these systems, numerous longitudinal modes do exist ${ }^{51}$. Four wave mixing between different modes results in huge spectral broadening and could be described in terms of wave turbulence ${ }^{52-54}$. Four-wave mixing processes lead to a redistribution of energy from the central modes to side-bands, and some partial correlations between different longitudinal modes could arise in these processes. Indications of mode correlations in Raman fiber laser radiation is reported recently in $^{55}$. Moreover, dark and grey solitons can be generated in normal dispersion quasi-CW Raman fiber laser ${ }^{56}$. The origin of the correlations is unknown both in long lasers of conventional cavities and in random fibre lasers. 


\section{CONCULSION}

Thus in this work we suggested a new full numerical iterative approach that allow one to describe spectral, temporal and coherent properties of DFB fiber lasers based on Rayleigh scattering. The model is based on a set of generalized nonlinear Schrödinger equations for pump and Stokes waves averaged over micron-scale fiber inhomogeneities. Random distributed feedback taken into account has proper power resulted from balance model and spectrum obtained for counter-propagating wave in previous iteration. Calculated generation power and its longitudinal distribution as well as optical spectrum are in good qualitative agreement with previous experimental results. It is shown that increasing the dispersion or decreasing the nonlinear coefficient leads to the narrower generation spectrum providing a possibility to spectral management of random DFB fiber laser generation. Temporal and statistical properties of radiation are also studied. The intensity statistics and intensity auto-correlation function reveal non-gaussian statistics of the random DFB fiber laser radiation what suggests presence of correlations in DFB laser radiation.

\section{REFERENCES}

[1] Turitsyn, S. K., Babin, S.A., El-Taher, A. E., Harper, P., Churkin, D. V., Kablukov, S. I., Ania-Castañón, J. D. , Karalekas, V., and Podivilov, E. V., "Random distributed feedback fibre laser," Nature Photonics 4, 231-235 (2010).

[2] Churkin, D. V., Babin, S. A., El-Taher, A. E. , Harper, P., Kablukov, S. I., Karalekas, V., Ania-Castañón, J. D., Podivilov, E.V., and Turitsyn, S. K., "Raman fiber lasers with a random distributed feedback based on Rayleigh scattering," Phys. Rev. A 82, 033828 (2010).

[3] Vatnik, I. D., Churkin, D. V., Babin, S. A., and Turitsyn, S. K. "Cascaded random distributed feedback Raman fiber laser operating at $1.2 \mu \mathrm{m}$," Opt. Express 19, 18486-18494 (2011).

[4] Teng, R. , Ding, Y., and Chen, L. "Random fiber laser operating at 1,115 nm," Applied Physics B, 111, 169$172,(2013)$.

[5] Zhang, W. L., Rao, Y. J., Zhu, J. M., Yang, Z. X., Wang, Z. N., and Jia, X. H. "Low threshold 2nd-order random lasing of a fiber laser with a half-opened cavity.," Optics express 20, 14400-5 (2012).

[6] Pinto, A. M. R., Frazão, O., Santos, J. L., and Lopez-Amo, M. "Multiwavelength fiber laser based on a photonic crystal fiber loop mirror with cooperative Rayleigh scattering," Appl. Phys. B 99, 391-395 (2010).

[7] Zhang, W. L., Zhu, Y. Y., Rao, Y. J., Wang, Z. N., Jia, X. H., and Wu, H. "Random fiber laser formed by mixing dispersion compensated fiber and single mode fiber," Optics Express 21, 8544 (2013).

[8] Sarmani, A. R., Abu Bakar, M. H. , Bakar, A. A. A., Adikan, F. R. M., and Mahdi, M. A. "Spectral variations of the output spectrum in a random distributed feedback Raman fiber laser," Optics express 19, 14152-14159 (2011).

[9] Sarmani, A. R., Abu Bakar, M. H., Adikan, F. R. M., and Mahdi, M. A. "Laser parameter variations in a rayleigh scattering-based raman fiber laser with single fiber bragg grating reflector." IEEE Photonics Journal 4, 461-466 (2012).

[10]El-Taher, A. E., Harper, P., Babin, S. A., Churkin, D.V., Podivilov, E. V, Ania-Castañón, J. D., and Turitsyn, S. K., "Effect of Rayleigh-scattering distributed feedback on multiwavelength Raman fiber laser generation," Opt. Lett. 36, 130-132 (2011).

[11] Sugavanam, S., Tarasov, N., Shu, X., and Churkin, D. V. "Narrow-band generation in random distributed feedback fiber laser," Opt. Express 21(14), 16466-16472 (2013).

[12] Sugavanam, S., Yan, Z., Kamynin, V., Kurkov, A. S., Zhang, L. and Churkin, D. V. "Multiwavelength generation in a random distributed feedback fiber laser using an all fiber Lyot filter," Optics Express 22, 28392844 (2014).

[13] Sarmani, A., Zamiri, R., and Bakar, M. "Tunable Raman fiber laser induced by Rayleigh backscattering in an ultra-long cavity," Journal of the European Optical society, 11043, 4-7 (2011).

[14] Babin, S. A. , El-Taher, A. E., Harper, P., Podivilov, E. V., and Turitsyn, S. K. "Tunable random fiber laser," Phys. Rev. A 84, 021805 (2011).

[15] Pang, M., Xie, S., Bao, X., Zhou, D., Lu, Y., and Chen, L. "Rayleigh scattering-assisted narrow linewidth Brillouin lasing in cascaded fiber," Opt. Lett.37, 3129-3131 (2012).

[16] Rao, Y. J., Zhang, L. W., Zhu, J. M., Yang, Z. X., Wang, Z. N., and Jia, X. H. "Hybrid lasing in an ultra-long ring fiber laser," Opt. Express 20, 22563-22568 (2012). 
[17] Churkin, D. V., El-Taher, Vatnik, I. D., Ania-Castañón, J. D., Harper, P., Podivilov, E. V., Babin, S. A. and Turitsyn, S. K. "Experimental and theoretical study of longitudinal power distribution in a random DFB fiber laser," Opt. Express 20, 11178-11188 (2012).

[18] Nuño, J., Alcon-Camas, M., and Ania-Castanon, J. D. "RIN transfer in random distributed feedback fiber lasers," Opt. Express 20, 27376-27381 (2012).

[19] Pinto, A. M. R., Lopez-Amo, M.. Kobelke, J., and Schuster, K. "Temperature fiber laser sensor based on a hybrid cavity and a random mirror," J. Lightwave Technol. 30, 1168-1172 (2012).

[20] Pinto, A. M. R., Frazão, O., Santos, J. L., Lopez-Amo, M.., Kobelke, J. and Schuster, K. "Interrogation of a suspended-core Fabry-Perot temperature sensor through a dual wavelength Raman fiber laser," J. of Lightwave Technology 28, 3149-3155 (2010).

[21] Martins, H. F., Marques, M. B, and Frazão, O. "Temperature-insensitive strain sensor based on four-wave mixing using Raman fiber Bragg grating laser sensor with cooperative Rayleigh scattering," Applied Physics B 104, 957-960 (2011).

[22] Wiersma, D. "Disordered photonics," Nature Photonics 7, 188-196 (2013).

[23] Vatnik, I. D. , Churkin, D. V., and Babin, S. A. "Power optimization of random distributed feedback fiber lasers," Opt. Express 20, 28033-28038 (2012).

[24] Cao, H., Xu, J. Y., Zhang, D. Z., Chang, S.-H., Ho, S. T., Seelig, E. W., Liu, X., and Chang, R. P. H. "Spatial confinement of laser light in active random media," Phys. Rev. Lett. 84, 5584-5587 (2000).

[25] Mujumdar, S.,. Ricci, M , Torre, R., and Wiersma, D. S. "Amplified extended modes in random lasers," Phys. Rev. Lett. 93, 053903 (2004).

[26] Türeci, H. E., Ge, L., Rotter, S., and Stone, A. D. "Strong interactions in multimode random lasers," Science 320, 643-646 (2008).

[27] Fallert, J., Dietz, R. J. B., Sartor, J., Schneider, D., Klingshirn, C., and Kalt, H. "Co-existence of strongly and weakly localized random laser modes," Nature Photon. 3, 279-282 (2009).

[28] Stano, P.and Jacquod, P. "Suppression of interactions in multimode random lasers in the Anderson localized regime," Nature Photon. 7, 66-71 (2013).

[29] Wang, Z., Jia, X., Rao, Y., Jiang, Y., and Zhang, W. "Novel long-distance fiber-optic sensing systems based on random fiber lasers," Proc. SPIE 8351, 835142-1-4 (2012).

[30] Vanneste, C., Sebbah, P., and Cao, H. "Lasing with resonant feedback in weakly scattering random systems." Phys. Rev. Lett. 98, 143902 (2007).

[31] Berger, G. A., Kempe, M., and Genack, A. Z. "Dynamics of stimulated emission from random media," Phys. Rev. E 56, 6118-6122 (1997).

[32] Jiang, X., and Soukoulis, C. M. “Time dependent theory for random lasers," Phys. Rev. Lett. 85, 70-73 (2000).

[33] Lü, J., Liu, J., Liu, H., Wang, K., and Wang, S. "Theoretical investigation on temporal properties of random lasers pumped by femtosecond-lasing pulses," Optics comm. 282, 2104-2109 (2009).

[34] Andreasen, J., and Cao, H. "Numerical study of amplified spontaneous emission and lasing in random media," Phys. Rev. A 82, 063835 (2010).

[35] Wu, X., Andreasen, J., Cao, H., and Yamilov, A. "Effect of local pumping on random laser modes in one dimension," Journ. Opt. Soc. Am. B 24, A26-A33 (2007).

[36] Xie, Y., and Liu, Z.A. "A new physical model on lasing in active random media," Phys. Lett. A 341, 339-344 (2005).

[37] Smirnov, S.V. and Churkin, D. V. "Modeling of spectral and statistical properties of a random distributed feedback fiber laser." Optics Eexpress, 21, 21236-21241 (2013).

[38] Agrawal, G. P. [Nonlinear fiber optics], Academic Press (2001).

[39] Preda, C. E., Ravet, G., Fotiadi, A. A., and Mégret, P."Iterative method for Brillouin fiber ring resonator," in CLEO/Europe 2011 Conference, OSA Technical Digest (Optical Society of America, 2011), paper CJ_P27.

[40] Turitsyn, S. K., Bednyakova, A. E., Fedoruk, M. P., Latkin, A. I., Fotiadi, A. A., Kurkov, A. S., and Sholokhov, E. "Modeling of CW Yb-doped fiber lasers with highly nonlinear cavity dynamics," Opt. Express 19, 12271230 (2011).

[41] Bednyakova, A. E., Gorbunov, O.A., Politko, M. O., Kablukov, S. I., Smirnov, S. V., Churkin, D. V., Fedoruk, M. P. and Babin, S. A. "Generation dynamics of the narrowband Yb-doped fiber laser," Opt. Express 21, 8177-8182 (2013).

[42] Churkin, D. V., Smirnov, S. V., and Podivilov, E. V. "Statistical properties of partially coherent cw fiber lasers," Opt. Lett. 35, 3288-3290 (2010). 
[43] Randoux, S., Dalloz, N., and Suret, P. "Intracavity changes in the field statistics of Raman fiber lasers," Opt. Lett. 36, 790-792 (2011).

[44] Churkin, D. V., Gorbunov, O. A., and Smirnov, S. V. "Extreme value statistics in Raman fiber lasers," Opt. Lett. 36, 3617-3619 (2011).

[45] Churkin, D. V., and Smirnov, S. V. "Numerical modelling of spectral, temporal and statistical properties of Raman fiber lasers," Opt. Commun. 285, 2154-2160 (2012).

[46] Smith, R. G. "Optical power handling capacity of low loss optical fibers as determined by stimulated Raman and Brillouin scattering," Appl. Opt. 11, 2489-2494 (1972).

[47]Fotiadi, A. A. and Kiyan, R. V. "Cooperative stimulated Brillouin and Rayleigh backscattering process in optical fiber," Opt. Lett. 23, 1805-1807 (1998).

[48] Ania-Castañón, J. D. "Quasi-lossless transmission using second-order Raman amplification and fibre Bragg gratings," Opt. Express 12, 4372-4377 (2004).

[49]Zervas, M. N. and Laming, R. I. "Rayleigh scattering effect on the gain efficiency and noise of Erbium-doped fiber amplifiers," IEEE Journ. of Quant. El. 31, 468-471 (1995).

[50] Mermelstein, M. D., Posey, Jr. R., Johnson, G. A. and Vohra, S. T. "Rayleigh scattering optical frequency correlation in a single-mode optical fiber," Opt. Lett. 26, 58-60 (2001).

[51]Babin, S. A., Karalekas, V. , Harper, P., Podivilov, E. V., Mezentsev, V. K., Ania-Castañón, J. D., and. K. Turitsyn, S. K."Experimental demonstration of mode structure in ultralong Raman fiber lasers," Opt. Lett. 32, 1135-1137 (2007).

[52] Babin, S. A., Churkin, D. V., Ismagulov, A. E., Kablukov, S. I., and Podivilov, E. V. "FWM-induced turbulent spectral broadening in a long Raman fibre laser," J. Opt. Soc. Am. B 24, 1729-1738 (2007).

[53] Babin, S. A., Churkin, D. V., Ismagulov, A. E., Kablukov, S. I., and Podivilov, E. V., "Spectral broadening in Raman fiber lasers," Optics letters 31, 3007-3009 (2006).

[54] Babin, S. A., Churkin, D. V., Ismagulov, A. E., Kablukov, S. I., and Podivilov, E. V. "Turbulence-induced square-root broadening of the Raman fiber laser output spectrum," Optics letters 33, 633-635 (2008).

[55] Vatnik, I.D., Gorbunov, O. A., and Churkin, D. V. "Narrow-band generation and mode correlations in a short Raman fibre laser." Laser Physics 24, 025103 (2014).

[56] Turitsyna, E. G., Smirnov, S. V., Sugavanam, S., Tarasov, N., Shu, X., Babin, S., Podivilov, E. V., Churkin, D. V., Falkovich, G., and Turitsyn, S. K. "The laminar-turbulent transition in a fibre laser," Natutre Photonics 7(10), 783-786 (2013). 